\title{
SPECTRAL METHOD FOR TIME DEPENDENT NAVIER-STOKES EQUATIONS
}

\author{
GABRIELLA BOGNÁR AND ZOLTÁN CSÁTI
}

Received 18 October, 2015

\begin{abstract}
The paper is focused on the numerical investigation of the Navier-Stokes equation applying a spectral method. A MATLAB code is developed and used for simulation. The incompressible two-dimensional flow in a square container called lid-driven cavity is tested as a benchmark problem. The streamlines, the horizontal and vertical velocity components at different Reynolds numbers are presented. There is significant agreement with the numerical results and those published in the literature.
\end{abstract}

2010 Mathematics Subject Classification: 34B10; 34B15

Keywords: Navier-Stokes equation, spectral method, MATLAB, lid-driven cavity, vortex

\section{INTRODUCTION}

Exact solution to the Navier-Stokes equation can be found only in very special cases. The most common numerical methods include finite difference (FDM), finite element (FEM) and finite volume methods (FVM). The advantage of the finite difference method is that it is easy to implement, while the other two are capable of handling complex domains and natural boundary conditions.

In this paper a spectral method is applied to provide a program in MATLAB for the solution of the Navier-Stokes equation. If the domain is simple and the solution is smooth enough, spectral methods, being high-order methods, are considered for their relatively low computational and memory demand, which is particularly important in fluid mechanics. However, their realization on a computer is more difficult than that of the previously mentioned three well-known methods.

A square cavity filled with incompressible Newtonian fluid is considered when the internal recirculating flows are generated by the motion of one containing wall. The top of the cavity is a plate that moves horizontally with constant velocity. The geometry is very simple, nevertheless the problem is of great scientific interest because it displays almost all fluid mechanical phenomena such as corner eddies, rotating vortices, instabilities, turbulence etc. This facilitates the comparison of results from experiments and computation. This problem is widely employed to evaluate numerical methods and to validate codes. 
Although it is a stationary problem at first sight, as the Reynolds number increases, the steady flow becomes unstable characterized by a Hopf bifurcation. The benchmark problem has been solved by many authors with different methods. Burggraf [6] was the first who considered the problem in 1966 and solved it with FDM. Bruneau et al. [5] started from the primitive variable formulation of the problem with solution by FDM combined with a multigrid solver. Vanka [17] applied a block-implicit multigrid method on the FDM discretized equations. The global nature of spectral methods causes the corner singularities to be even more detrimental to the convergence. Therefore, those singularities were removed by Botella and Peyret in [3]. Most techniques rely on the vorticity-stream function formulas, see for example Ghia et al. [9], Schreiber et al. [15] and Benjamin et al. [2]. Barragy and Carey used the $p$ version of FEM [1], while Marchi et al. [13] and Yapici at al. [19] the FVM. In [19] the authors even produced results for very high $(\mathrm{Re}=65000)$ Reynolds number.

Our aim is to provide a MATLAB program for the numerical solution of the incompressible Navier-Stokes equations. In order to test our code, one of the most famous benchmark problems, the lid-driven cavity task and its regularized version are solved and the achieved solutions are compared with results existing in the literature. Reliable error estimates are shown. Although, the steady solutions are compared, we achieve the steady state through the time dependent case.

\section{GOVERNING EQUATIONS}

The mathematical model of the motion of an incompressible, laminar, Newtonian fluid is characterized by the continuity equation and the Navier-Stokes equations. After nondimensionalization, their instationary form reads [12]

$$
\begin{aligned}
\frac{\partial \mathbf{v}}{\partial t}+(\mathbf{v} \cdot \nabla) \stackrel{\downarrow}{\mathbf{v}}+\nabla p & =\frac{1}{\operatorname{Re}} \Delta \mathbf{v}+\mathbf{f}, & & \mathbf{x} \in \Omega \subset \mathbb{R}^{3}, t \in[0, T] \\
\mathbf{v} \cdot \nabla & =0, & & \mathbf{x} \in \Omega \subset \mathbb{R}^{3}, t \in[0, T] .
\end{aligned}
$$

In the equations, $\mathbf{x}$ is the position vector of the current configuration, $\operatorname{Re}=v L / v$ is the Reynolds number, $v$ and $L$ are the characteristic velocity and length, $v=\mu / \rho_{0}$ is the kinematic viscosity, $\mu$ is the dynamic viscosity, $\rho_{0}$ is the density and the forcing term $\mathbf{f}$ is a function at least once differentiable with respect to the spatial coordinates. The quantity $p$ denotes the quotient of the pressure and constant $\rho_{0}$. These equations constitute the primary variable formulation of the problem.

Equations (2.1), (2.2) are considered under the initial condition

$$
\mathbf{v}=\mathbf{v}_{\mathbf{0}}, \quad \mathbf{x} \in \Omega \cup \partial \Omega, t=0
$$

and boundary conditions

$$
\begin{aligned}
& \mathbf{v}=\mathbf{v}^{D}, \quad \mathbf{x} \in \partial \Omega^{D}, \quad t \in[0, T], \\
& \sigma \cdot \mathbf{n}=\mathbf{f}^{N}, \quad \mathbf{x} \in \partial \Omega^{N}, t \in[0, T],
\end{aligned}
$$


where $\sigma$ is the Cauchy stress tensor and $\mathbf{n}$ is the outward normal vector of the surface. An initial velocity field is compatible if $\nabla \cdot \mathbf{v}_{\mathbf{0}}=0$ and $\mathbf{v}_{\mathbf{0}} \cdot \mathbf{n}=\mathbf{v}^{D} \cdot \mathbf{n}$. There is no initial and boundary condition for the pressure, but it is not necessary because in (2.1) the gradient of $p$ is involved. Therefore, the pressure is determined only up to a constant in space. For the solution to be unique, the mean value of the pressure is taken to be 0 :

$$
\int_{\Omega} p \mathrm{~d} V=0
$$

and is taken into account with a Lagrange multiplier.

There is no method that could be systematically applied and would be the best in the solution of (2.1)-(2.2). The problem is difficult because the differential equation (2.1) is nonlinear even if the fluid is Newtonian. The fundamental difficulty is that the pressure is not present in the continuity equation, which results in a zero-matrix block in the coefficient matrix of the discretized system, making it singular [12]. It applies to both the finite element and the spectral methods. Several methods exist to avoid this case. To circumvent the LBB inf-sup condition, $\mathbb{P}_{N}-\mathbb{P}_{N-2}$ approximation is often used (see e.g., [8]). Another method is to find the nodal variables (velocity and pressure) at different nodes, called the staggered grid approach. Its spectral implementation is cumbersome mainly for inhomogeneous boundary conditions [16]. The widely applied projection method introduced by Chorin [7] consists of two steps. At first, a provisional velocity field is calculated, which satisfies the boundary conditions but not the continuity equation. Then, in the second step, this velocity field is projected to the divergence-free velocity field satisfying (2.2). Weinan and Liu [18] introduced the gauge formalism, which eliminates the pressure from the equations, therefore no artificial boundary condition is required for the pressure [18].

In two dimensions the vorticity-stream function formalism is especially advantageous when instead of three unknowns, only two remains: the vorticity $\omega$ and the stream function $\psi$, for which

$$
u=\frac{\partial \psi}{\partial y}, \quad v=-\frac{\partial \psi}{\partial x}, \quad \omega=\frac{\partial v}{\partial x}-\frac{\partial u}{\partial y} .
$$

Here $\mathbf{v}=u \mathbf{i}+v \mathbf{j}$ and (2.2) is fulfilled by (2.6). The remaining two scalar equations are [14]

$$
\begin{gathered}
\Delta \psi+\omega=0, \\
\frac{\partial \omega}{\partial t}+\mathbf{v} \cdot \nabla \omega=\frac{1}{\operatorname{Re}} \Delta \omega+F,
\end{gathered}
$$

where $F=(\nabla \times \mathbf{f}) \cdot \mathbf{k}$. In case of Dirichlet boundary condition (2.4), the boundary conditions for the differential equations (2.7), (2.8) are the following

$$
\psi=-\int_{s_{0}}^{s} \mathbf{v}^{D} \cdot \mathbf{n} \mathrm{d} \hat{s}
$$




$$
\frac{\partial \psi}{\partial n}=\mathbf{v}^{D} \cdot \mathbf{t}
$$

where $s_{0}$ is a fixed point and $s(x, y)$ is a variable point of the boundary curve. The difficulty in the solution is that there are two boundary conditions for $\psi$ and no for $\omega$.

\section{SPeCtral METHOD}

In the lack of periodicity, we use the Chebyshev-Chebyshev spectral collocation method, i.e., the approximation of a function $u(x, y)$ is carried out by the tensor product of Chebyshev polynomials. The error of the spectral approximation is $\mathcal{O}\left(N^{-m}\right)$, where $m$ denotes the number of continuous derivatives.

\subsection{Discretization}

If $u(x, y)$ is approximated in the Lagrange (nodal) basis as

$$
p_{N} u(x, y)=\sum_{i=0}^{N} \sum_{j=0}^{N} u_{i, j} \ell_{i}(x) \ell_{j}(y),
$$

then the first order partial derivatives are calculated as [11]

$$
\begin{aligned}
& \frac{\partial u\left(x_{i}, y_{j}\right)}{\partial x}=\sum_{k, l=0}^{N} u_{k, l} \ell_{k}^{\prime}\left(x_{i}\right) \ell_{l}\left(y_{j}\right)=\sum_{k=0}^{N} u_{k, j} D_{i k}=\mathbf{D} \mathbf{U}, \\
& \frac{\partial u\left(x_{i}, y_{j}\right)}{\partial y}=\sum_{k, l=0}^{N} u_{k, l} \ell_{k}\left(x_{i}\right) \ell_{l}^{\prime}\left(y_{j}\right)=\sum_{l=0}^{N} u_{i, l} D_{j l}=\mathbf{U D}^{T},
\end{aligned}
$$

where $\mathbf{D}$ is the (first) differentiation matrix and $\mathbf{U}$ contains the discrete unknowns at the Chebyshev collocation points. The higher order derivatives are approximated likewise. The matrix-vector product is not the only way to perform spectral differentiation. For larger $N$, the discrete Fourier transform is more effective. The differentiation matrices are indefinite, non-symmetric, dense matrices with large condition number. Therefore, either a preconditioned iterative or a dense direct solver is used to solve the system of algebraic equations.

During the determination of the numerical solution to an initial-boundary value problem (IBVP), one can choose to discretize first in time and then in space (Rothe's method) or vice versa (method of lines). Here, the first one is applied, where the solution of time-independent partial differential equations is necessary in each time step. From many existing temporal discretization schemes, the semi-implicit backward Euler (SIBE) and the semi-implicit second order Adams-Bashforh/backward differentiation (AB/BDI2) methods are implemented. These schemes have been experienced to be robust for incompressible fluid flow problems [14]. Applying the 
SIBE semi-discretization method, approximation of the differential equation is

$$
\frac{\partial u}{\partial t}=L(u)+N(u)
$$

where $L$ is a linear, $N$ is a nonlinear spatial differential operator and

$$
\frac{u^{n+1}-u^{n}}{\Delta t}=L\left(u^{n+1}\right)+N\left(u^{n}\right)
$$

whereas the $\mathrm{AB} / \mathrm{BDI} 2$ scheme is as follows

$$
\frac{3 u^{n+1}-4 u^{n}+u^{n-1}}{2 \Delta t}=L\left(u^{n+1}\right)+2 N\left(u^{n}\right)-N\left(u^{n-1}\right) .
$$

The time step $\Delta t$ is taken constant. The AB/BDI2 is a two-step method, hence it is necessary to be started. In [14], it is proved that a first order method is appropriate for starting this second order method with the second order accuracy retained. So, we start it with SIBE. The semi-discrete system is then

$$
\begin{gathered}
\Delta \omega^{n+1}-\sigma \omega^{n+1}=f^{n}, \\
\Delta \psi^{n+1}+\omega^{n+1}=0,
\end{gathered}
$$

where

for SIBE and

$$
\sigma=\frac{\operatorname{Re}}{\Delta t}, \quad f^{n}=\operatorname{Re}\left(\mathbf{v}^{n} \cdot \nabla \omega^{n}-\frac{\omega^{n}}{\Delta t}\right)
$$

$$
\sigma=\frac{3 \operatorname{Re}}{2 \Delta t}, \quad f^{n}=\operatorname{Re}\left(2 \mathbf{v}^{n} \cdot \nabla \omega^{n}-\mathbf{v}^{n-1} \cdot \nabla \omega^{n-1}+\frac{-4 \omega^{n}+\omega^{n-1}}{2 \Delta t}\right)
$$

for $\mathrm{AB} / \mathrm{BDI} 2$, respectively. The first equation of (3.6) is solved for $\omega^{n+1}$. Then, $\psi^{n+1}$ can be determined from the second equation. It means that at every time step, the solution to a Helmholtz and to a Poisson equation are sought. The problem with the solution of equations (3.6) with boundary conditions (2.9)-(2.10) is tackled with the so-called influence matrix method. This method utilizes the linear nature of the Helmholtz and the Poisson equations and is described in detail in [14].

At this level, the numerical solution to the original IBVP is traced back to the solution of the Helmholtz equation. For simplicity, let us regard the $(-1,1) \times(-1,1)$ square domain. Choosing the collocation points to be the tensor product of the extrema of the Chebyshev polynomials of the first kind:

$$
\left(x_{i}, y_{j}\right)=\left(-\cos \frac{i \pi}{N},-\cos \frac{j \pi}{N}\right), \quad i, j=0, \ldots, N,
$$

the corresponding discrete form of the boundary value problem

$$
\begin{gathered}
\frac{\partial^{2} u}{\partial x^{2}}+\frac{\partial^{2} u}{\partial y^{2}}-\sigma u=f, \quad(x, y) \in \Omega, \quad \sigma>0 \\
u=g, \quad(x, y) \in \Gamma=\partial \Omega,
\end{gathered}
$$


can be formulated as

$$
\mathbf{D}^{(2)} \mathbf{U}+\mathbf{U D}^{(2)^{T}}-\sigma \mathbf{U}=\mathbf{H} .
$$

This matrix-matrix product can be written as the usual form of linear systems using the Kronecker product [10]:

$$
\left(\mathbf{I} \otimes \mathbf{D}^{(2)}+\mathbf{D}^{(2)} \otimes \mathbf{I}-\sigma \mathbf{I} \otimes \mathbf{I}\right) \mathbf{u}=\mathbf{g},
$$

where $\mathbf{I}$ is the $N$-by- $N$ identity matrix, $\mathbf{u}=\operatorname{vec}(\mathbf{U})$ and $\mathbf{g}=\operatorname{vec}(\mathbf{H})$.

For some kinds of differential operators, such as the Helmholtz operator, partial or full diagonalization remedies the large storage and computational demand of the coefficient matrix in (3.11). Since the eigenvalues of $\mathbf{D}^{(2)}$ are linearly independent, it can be diagonalized such as

$$
\mathbf{D}^{(2)}=\mathbf{P} \Lambda \mathbf{P}^{-1}
$$

where $\Lambda$ is a diagonal matrix containing the eigenvalues of $\mathbf{D}^{(2)}$ in its main diagonal and the columns of $\mathbf{P}$ contain the eigenvectors of $\mathbf{D}^{(2)}$. The diagonalization has to be performed only once at the preprocessing stage, not at every time step, making it an attractive alternative scheme.

\subsection{Stopping criterion, error estimation}

The stationary solution is obtained as the limit of the solution to the time-dependent problem. The stopping criterion is the relative $L_{2}$ error, i.e. for a function $u(\mathbf{x})$ :

$$
E_{N}(u)=\frac{\left\|u-u_{N}\right\|_{2}}{\|u\|_{2}} \approx \frac{\left\|u_{N}^{n+1}-u_{N}^{n}\right\|_{2}}{\left\|u_{N}^{n+1}\right\|_{2}},
$$

where $u$ is the exact, $u_{N}$ is the approximate solution.

Several rules based on theories and numerical experiences help us to decide whether the numerical solution is appropriate or not [4]. For a univariate function the following steps are considered

- perform the calculation on finer grids,

- the trailing few Chebyshev coefficients should decrease monotonically in absolute value,

- the magnitude of the truncation error is about the magnitude of the last retained spectral coefficient:

$$
E_{T}(N) \approx \mathcal{O}\left(\left|a_{N}\right|\right) .
$$

In two dimensions, due to the tensor product construction, the same is applicable, but the $a_{m n}$ Chebyshev coefficients have to be taken as a function of $m+n$.

The described method was implemented in MATLAB, version R2011a and is available from

http://www . mathworks.com/matlabcentral/fileexchange/

47183-navier-stokes-solver. 
The obtained functions are modular and realize different visualization techniques. Two-dimensional barycentric Lagrange-interpolation and some utility functions are also included.

\section{NUMERICAL TEST}

The code is tested on the classic fluid flow, lid-driven cavity problem and on its regularized version.

\subsection{Lid-driven cavity}

We investigate the flow in a rectangular container called lid-driven cavity. The flow is induced by the tangential movement of the upper wall (lid). A $2 \times 2$ square cavity with a constant moving plate on its upper edge is chosen to do the simulations. The left, right and lower wall of the square represent physical walls. Thus, no slip condition is applied for the test. For the upper boundary the velocity is set to constant. Due to the simple geometry and boundary conditions the lid-driven cavity is an ideal system for investigating flow phenomena like corner eddies, vortices, instability ad transition to turbulence. It is also used as a benchmark for numerical codes.

Since the fluid is viscous, it starts circulating. Note, that the velocity field is not continuous at the upper left and upper right hand corner, which deteriorates the convergence severely. The initial velocity is taken $\mathbf{v}_{\mathbf{0}}=0$, which satisfies the compatibility conditions. Numerical calculations are performed for different values of Reynolds numbers. For $\mathrm{Re}=200$, choosing the time step $\Delta t=0.1$, the stopping criterion to $10^{-9}$, the stationary solution is achieved after 978 iterations using $N=20$-th order polynomials in both direction. Figure 1 shows the result with streamlines.

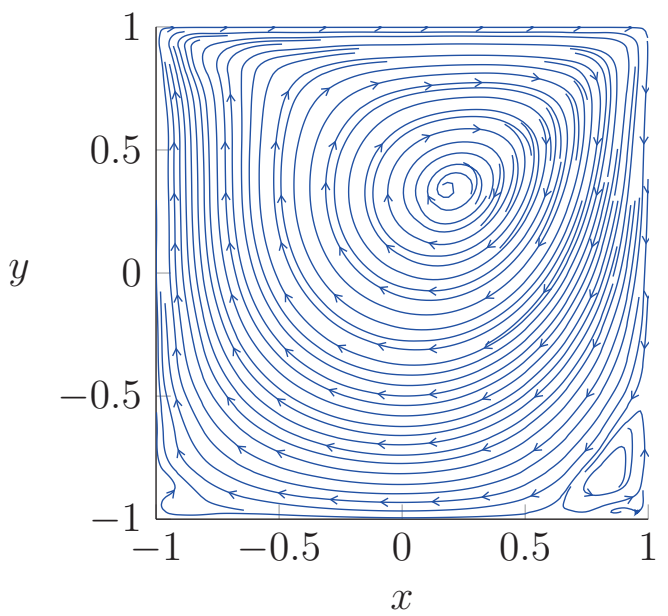

FIGURE 1. Streamlines, $\operatorname{Re}=200$ 
TABLE 1. Velocities at the center for $\mathrm{Re}=0.01$

\begin{tabular}{lcccc}
\hline & \multicolumn{3}{c}{ our calculation for different $N$} & \multirow{2}{*}{ from paper [13] } \\
\cline { 2 - 5 }$N$ & 10 & 20 & 50 & \\
\hline$u(0,0)$ & $-2.0541855 \mathrm{e}-1$ & $-2.0519521 \mathrm{e}-1$ & $-2.0519168 \mathrm{e}-1$ & $-2.05191715 \mathrm{e}-1$ \\
\hline$v(0,0)$ & $6.4550503 \mathrm{e}-6$ & $6.3644848 \mathrm{e}-6$ & $6.3676966 \mathrm{e}-6$ & $6.3677058 \mathrm{e}-6$ \\
\hline
\end{tabular}

If the Reynolds number is large, corresponding to small viscosity for fixed velocity, the differential equation becomes stiff and extremely small time steps are needed to maintain stability. To ensure the stability of the time discretization schemes, continuation is used. The continuation parameter is the Reynolds number, meaning that the high Reynolds number flows are simulated through small steps in Re. Tables 1-3 report the calculated velocity components at the center of the domain showing good agreement with results of [13]. The digits which agree are indicated by boldface. [htbp] As the Reynolds number increases, the corner singularities have a more and

TABLE 2. Velocities at the center for $\operatorname{Re}=100$

\begin{tabular}{lcccc}
\hline & \multicolumn{3}{c}{ our calculation for different $N$} & \multirow{2}{*}{ from paper [13] } \\
\cline { 2 - 5 }$N$ & 10 & 20 & 50 & \\
\hline$u(0,0)$ & $-2.0851006 \mathrm{e}-1$ & $-2.0912834 \mathrm{e}-1$ & $-2.0914913 \mathrm{e}-1$ & $-2.09149143 \mathrm{e}-1$ \\
\hline$v(0,0)$ & $5.7673833 \mathrm{e}-2$ & $5.7505097 \mathrm{e}-2$ & $5.7536504 \mathrm{e}-2$ & $5.7536559 \mathrm{e}-2$ \\
\hline
\end{tabular}

more significant effect on the convergence, as it is exhibited in Tables 1-3.

In Figs. 2-5 velocity profiles $u, v$ are plotted for different positions $x=$ const. and $y=$ const. The $x$ components of the velocity for $\mathrm{Re}=400$ as a function of $y$ are depicted on Fig. 3 and Fig. 4. Figures of the velocity components $u=u(x)$ for fixed $y$ and $u=u(y)$ for fixed $x$ for $\operatorname{Re}=400$ as a function of $x$ are depicted on Fig. 3 and Fig. 5.

TABLE 3. Velocities at the center for $\operatorname{Re}=400$

\begin{tabular}{lcccc}
\hline & \multicolumn{3}{c}{ our calculation for different $N$} & \multirow{2}{*}{ from paper [13] } \\
\cline { 2 - 5 }$N$ & 10 & 20 & 50 & \\
\hline$u(0,0)$ & $-9.5450522 \mathrm{e}-2$ & $-1.1534413 \mathrm{e}-1$ & $-1 . \mathbf{1 5 0 5 3 4 9 \mathrm { e } - 1}$ & $-1.15053628 \mathrm{e}-1$ \\
\hline$v(0,0)$ & $6.7025081 \mathrm{e}-2$ & $5.2218832 \mathrm{e}-2$ & $5.2058066 \mathrm{e}-2$ & $5.2058082 \mathrm{e}-2$ \\
\hline
\end{tabular}




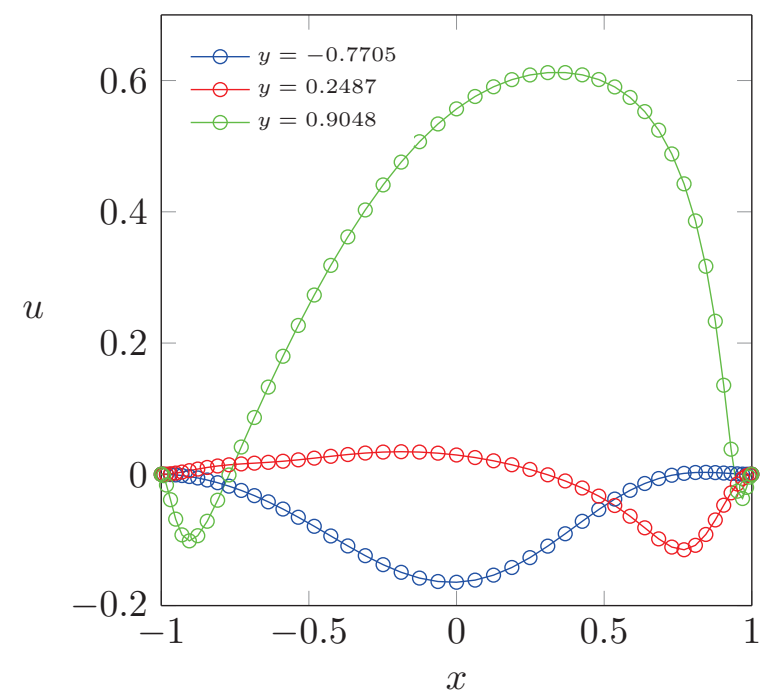

FIGURE 2. $u$ velocity profiles at different $y$ cross sections, $\operatorname{Re}=400$

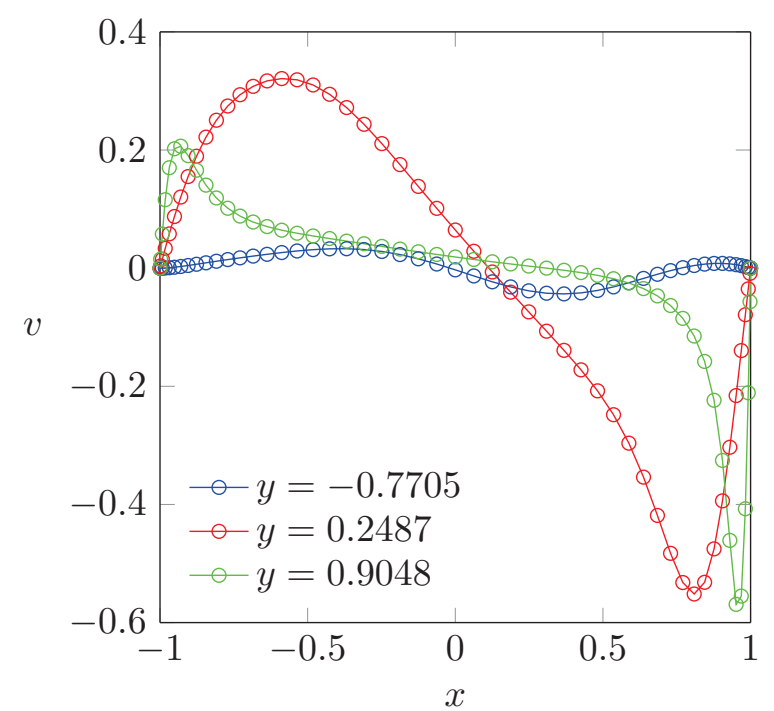

FIGURE 3. $v$ velocity profiles at different $y$ cross sections, $\mathrm{Re}=400$ 


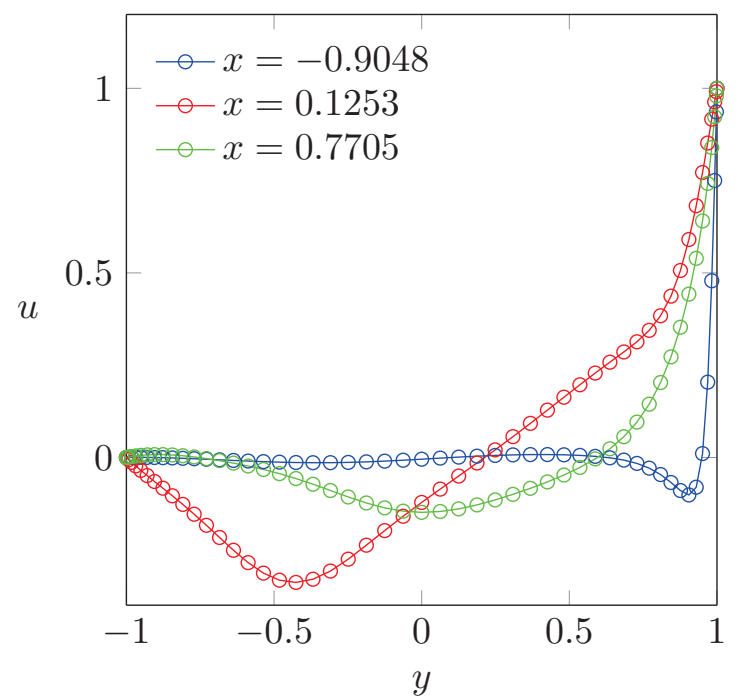

FIGURE 4. $u$ velocity profiles at different $x$ cross sections, $\operatorname{Re}=400$

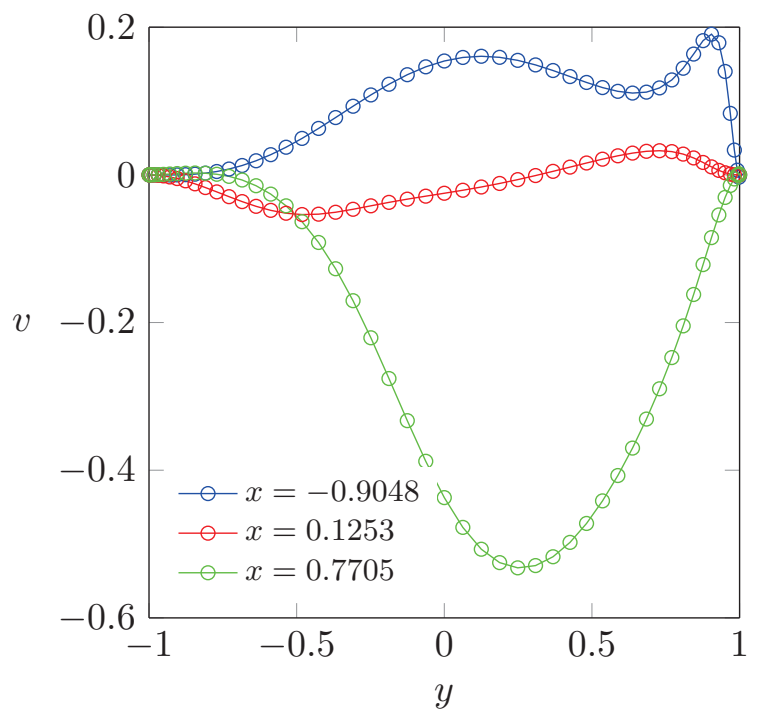

FIGURE 5. $v$ velocity profiles at different $x$ cross sections, $\operatorname{Re}=400$

For $\operatorname{Re}=400$, we need a smaller time step and more iterations, but the numerical solution can be obtained (see Fig. 6). 


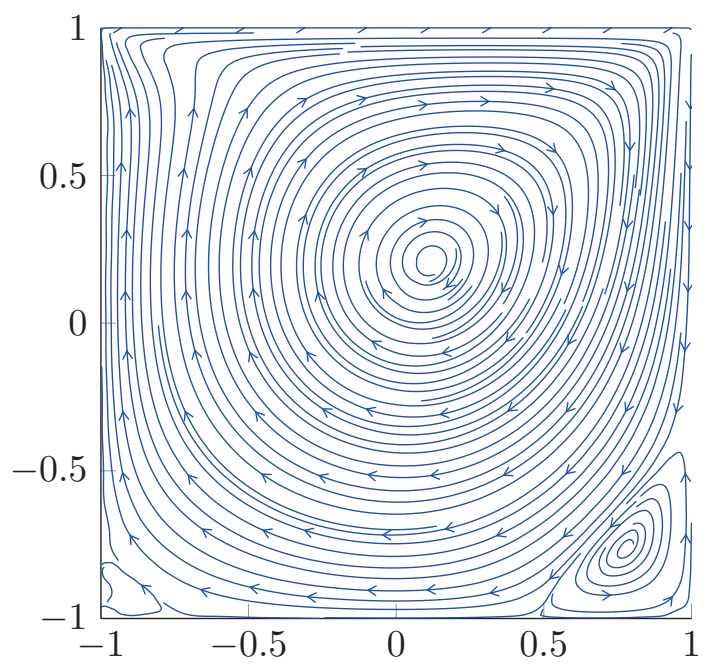

FIgURE 6. Streamlines, $\operatorname{Re}=400$

\subsection{Regularized lid-driven cavity}

The corner singularities are important issues in the flow field. The $y$ component of the velocity is required to be 1 on the lid at $y=1$ and zero at $x=-1, x=1$, therefore boundary condition is discontinuous at the upper corners. Removing the corner singularities (regularization) by setting the velocity of the lid to $u(x, 1)=$ $\left(x^{2}-1\right)^{2}$ results in a much higher convergence rate. The computations are repeated with $N=25$ and the settings of the previous subsection and the streamlines are depicted in Figure 7.

With the increase of $\mathrm{Re}$, the size of the secondary vortices in the corners is growing, while the primary vortex is moving towards the center of the cavity. Figure 8 suggests that the computations are correct because the Chebyshev coefficients are monotonically decreasing for both the primary $(\psi)$ and the derived $(u)$ variables. The spectral accuracy has been achieved with the application of regularization. We were able to raise the Reynolds number up to $\mathrm{Re}=8500$ (see Fig. 9).

\section{CONCLUSiONS}

In this work accurate numerical solutions are provided to the two-dimensional, incompressible, laminar Navier-Stokes equations by our MATLAB code. The LBB condition is eliminated by the vorticity-stream function formalism. Two types of time discretizations are built into the program. The barycentric Lagrange interpolation enables us to create the solution on a denser mesh in a stable way. The correctness of the program is tested on a fundamental benchmark problem, the lid-driven cavity flow problem. The achieved solutions are compared with results available in the 


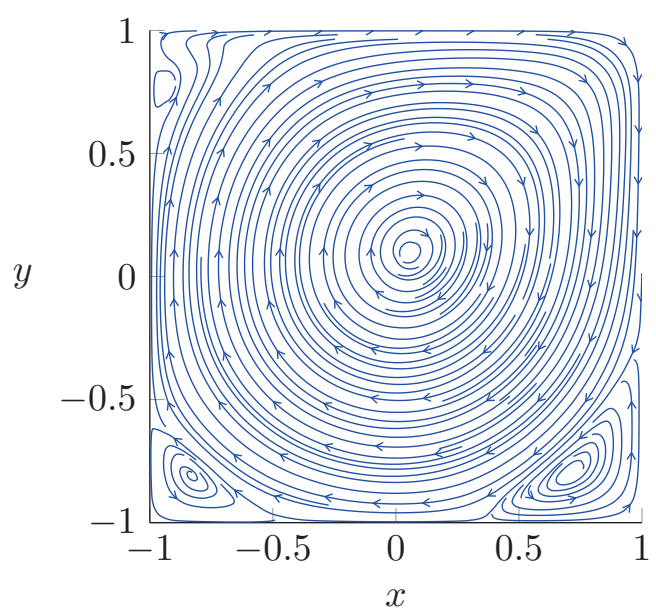

FIGURE 7. Streamlines, regularized cavity, $\mathrm{Re}=2000$

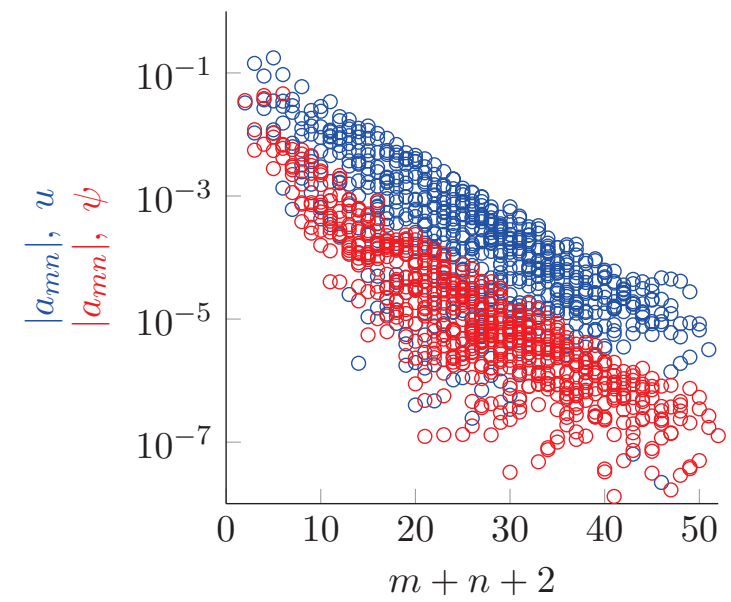

FIGURE 8. Spectral coefficients, regularized cavity, $R e=2000$

literature [13]. As the examples showed, the applied spectral method provides a feasible alternative to the existing methods. Opposite to the methods described in the introduction, our program can solve the instationary Navier-Stokes equations.

Since many problems in real life can be reduced to an axisymmetric 2D task, further improvements can be done by turning to a more general domain using domain decomposition methods and the support of non-Newtonian constitutive laws would also be beneficial. 


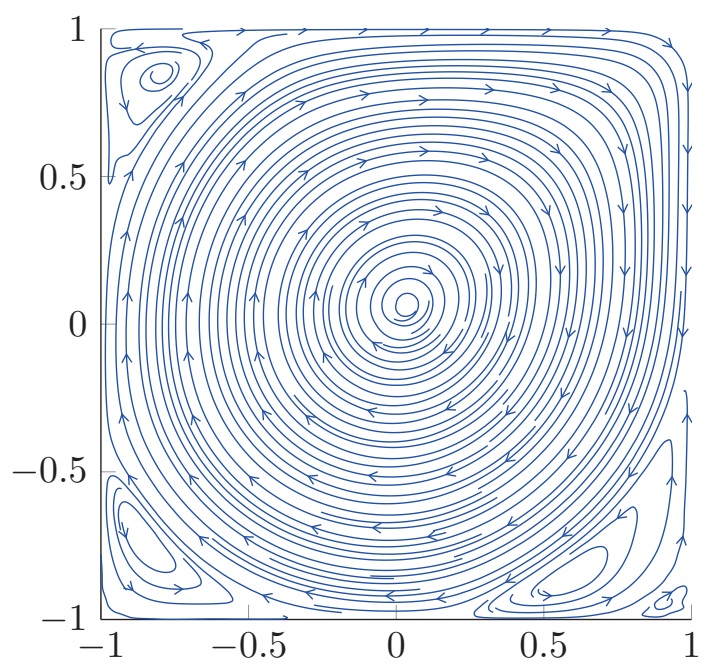

FIGURE 9. Streamlines, regularized cavity, $\operatorname{Re}=8500$

\section{ACKNOWLEDGEMENT}

The research work presented in this paper is based on the results achieved within the TÁMOP-4.2.1.B-10/2/KONV-2010-0001 project and carried out as part of the TÁMOP-4.1.1.C-12/1/KONV-2012-0002 "Cooperation between

higher education, research institutes and automotive industry" project in the framework of the New Széchenyi Plan. The realization of this project is supported by the Hungarian Government, by the European Union, and co-financed by the European Social Fund.

\section{REFERENCES}

[1] E. Barragy and G. F. Carey, "Stream function-vorticity driven cavity solution using p finite elements," Computers \& Fluids, vol. 26, no. 5, pp. 453-468, 1997, doi: 10.1016/S00457930(97)00004-2.

[2] A. S. Benjamin and V. E. Denny, "On the convergence of numerical solutions for 2-D flows in a cavity at large Re," Journal of Computational Physics, vol. 33, no. 3, pp. 340-358, 1979, doi: 10.1016/0021-9991(79)90160-8.

[3] O. Botella and R. Peyret, "Benchmark spectral results on the lid-driven cavity flow," Computers \& Fluids, vol. 27, no. 4, pp. 421-433, 1998, doi: 10.1016/S0045-7930(98)00002-4.

[4] J. P. Boyd, Chebyshev and Fourier Spectral Methods, ser. Dover Books on Mathematics. Dover Publications, 2000.

[5] C.-H. Bruneau and M. Saad, "The 2D lid-driven cavity problem revisited," Computers \& Fluids, vol. 35, no. 3, pp. 326-348, 2006, doi: 10.1016/j.compfluid.2004.12.004.

[6] O. R. Burggraf, "Analytical and numerical studies of the structure of steady separated flows," Journal of Fluid Mechanics, vol. 24, pp. 113-151, 1966, doi: 10.1017/S0022112066000545. 
[7] A. J. Chorin, "Numerical solution of the Navier-Stokes equations," Math. Comp., vol. 22, no. 6, pp. 745-762, 1968, doi: 10.1090/S0025-5718-1968-0242392-2.

[8] M. O. Deville, P. F. Fischer, and E. H. Mund, High-Order Methods for Incompressible Fluid Flow, 1st ed., ser. Cambridge Monographs on Applied and Computational Mathematics. Cambridge University Press, 2002, no. 9, doi: 10.1017/CBO9780511546792.

[9] U. Ghia, K. N. Ghia, and C. T. Shin, "High-Re solutions for incompressible flow using the NavierStokes equations and a multigrid method," Journal of Computational Physics, vol. 48, no. 3, pp. 387-411, 1982, doi: 10.1016/0021-9991(82)90058-4.

[10] R. A. Horn and C. R. Johnson, Topics in Matrix Analysis, 1st ed. Cambridge University Press, 1994.

[11] D. A. Kopriva, Implementing Spectral Methods for Partial Differential Equations: Algorithms for Scientists and Engineers, ser. Scientific Computation. Springer Netherlands, 2009. doi: 10.1007/978-90-481-2261-5.

[12] B. Q. Li, Discontinuous Finite Elements in Fluid Dynamics and Heat Transfer, ser. Computational Fluid and Solid Mechanics. Springer, 2006, vol. 5.

[13] C. H. Marchi, R. Suero, and L. Araki, "The Lid-Driven Square Cavity Flow: Numerical Solution with a 1024×1024 Grid," Journal of the Brazilian Society of Mechanical Sciences and Engineering, vol. 31, no. 3, pp. 186-198, 2009, doi: 10.1590/S1678-58782009000300004.

[14] R. Peyret, Spectral Methods for Incompressible Viscous Flow, ser. Applied Mathematical Sciences. Springer, 2001, vol. 148.

[15] R. Schreiber and H. B. Keller, "Driven cavity flows by efficient numerical techniques," Journal of Computational Physics, vol. 49, no. 2, pp. 310-333, 1983, doi: 10.1016/0021-9991(83)90129-8.

[16] M. R. Schumack, W. W. Schultz, and J. P. Boyd, "Spectral Method Solution of the Stokes Equations on Nonstaggered Grids," J. Comput. Phys., vol. 94, no. 1, pp. 30-58, 1991, doi: 10.1016/0021-9991(91)90136-9.

[17] S. P. Vanka, "Block-implicit multigrid solution of Navier-Stokes equations in primitive variables," Journal of Computational Physics, vol. 65, no. 1, pp. 138-158, 1986, doi: 10.1016/00219991(86)90008-2.

[18] E. Weinan and J.-G. Liu, "Gauge Method for Viscous Incompressible Flows," Commun. Math. Sci., vol. 1, no. 2, pp. 317-332, 2003, doi: 10.4310/CMS.2003.v1.n2.a6.

[19] K. Yapici and Y. Uludag, "Finite volume simulation of 2-D steady square lid driven cavity flow at high Reynolds numbers," Brazilian Journal of Chemical Engineering, vol. 30, no. 4, pp. 923-937, 2013, doi: 10.1590/S0104-66322013000400023.

\section{Authors' addresses}

\section{Gabriella Bognár}

University of Miskolc, Institute of Machine and Product Design, Miskolc-Egyetemváros, 3515

Miskolc, Hungary

E-mail address: v.bognar.gabriella@uni-miskolc.hu

\section{Zoltán Csáti}

University of Miskolc, Institute of Machine and Product Design, Miskolc-Egyetemváros, 3515 Miskolc, Hungary

E-mail address: machcsz@uni-miskolc.hu 